\title{
Fluorescent Nanodiamonds as Versatile Intracellular Temperature Sensors
}

\author{
Evgenii Glushkov*, Vytautas Navikas ${ }^{a}$, and Aleksandra Radenovic ${ }^{a}$
}

\begin{abstract}
Temperature is a widely known phenomenon, which plays an extremely important role in biological systems. Its behavior on the macro-scale has been quite well investigated and understood, thanks to the availability of reliable and precise thermometers such as thermocouples and infrared cameras. However, temperature measurements on the subcellular scale present an ongoing challenge due to the absence of universal nanoscale temperature sensors. Recent work on fluorescent nanodiamonds has revealed their unique ability to measure temperature with high spatial and temporal resolution, of particular importance in the intracellular environment. This review summarizes recent progress in the field and highlights the future directions for intracellular temperature sensing using fluorescent nanodiamonds.
\end{abstract}

Keywords: Fluorescence $\cdot$ Intracellular $\cdot$ Nanodiamonds $\cdot$ Sensing $\cdot$ Temperature

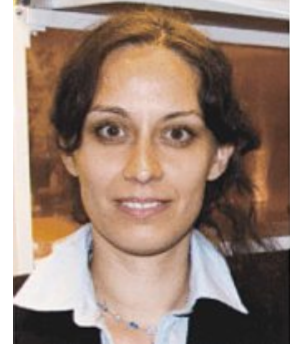

Aleksandra Radenovic received her master's degree in physics from the University of Zagreb in 1999 before joining Professor Giovanni Dietler's Laboratory of Physics of Living Matter in 2000 at University of Lausanne. There she earned her Doctor of Sciences degree in 2003. In 2003 she was also awarded a research scholarship for young researchers from the Swiss Foundation for Scientific Research which allowed her to spend three years as postdoctoral fellow at the University of California, Berkeley (2004-2007). Before joining EPFL as Assistant Professor in 2008 she spent six months at NIH and Janelia Farm. In 2010 she received the ERC starting grant and in 2015 a SNSF Consolidator grant. Her group is interested in using novel nanomaterials and single molecule experimental techniques to study fundamental questions in molecular and cell biology.

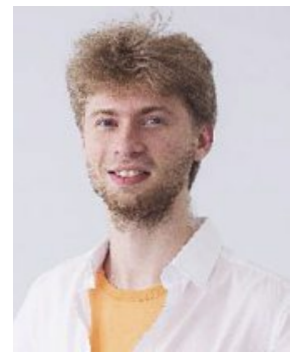

Evgenii Glushkov did his undergraduate studies at Moscow Institute of Physics and Technology (MIPT) working on both theoretical and experimental aspects of superconducting quantum systems and obtaining his Master degree with distinction in July, 2016. After that he was accepted to the EPFL doctoral school and joined the Laboratory of Nanoscale Biology to do his $\mathrm{PhD}$ under the supervision of Prof. Aleksandra Radenovic. The goal of Evgenii's project is to build intracellular sensors based on fluorescent nanodiamonds and use them to explore the cell's environment. This project is a part of the National Center of Competence in Research Bio-Inspired Materials, funded by the Swiss National Science Foundation. His other research interests include super-resolution microscopy, 2D materials and integrated photonics.

\section{Introduction}

No matter how ordinary and straightforward the notion of temperature might seem to us nowadays, it first appeared only around 300 years ago, when Galileo constructed his first thermoscope - a device capable of measuring relative temperature changes. ${ }^{[1]}$ Although the first thermoscopes were highly inaccurate, little progress was made in the years following his invention. Only in the $18^{\text {th }}$ century was a common temperature scale introduced, based on the work of Fahrenheit, Celsius, and Linnaeus. ${ }^{[2]}$ It took one more century for thermometers to find widespread use in medicine, stimulated by the invention of the clinical thermometer by Carl Wunderlich, which remained a standard tool for more than 130 years before being replaced by a thermocouple-based electronic thermometer.

While instruments to measure temperature at the macro-scale have developed considerably in the last few decades, measuring temperature with high resolution at the scale of micro- and nanometers remains challenging. ${ }^{[3,4]}$ The quest to create such a nanoscale thermometer is not only interesting from a fundamental point of view, but also for applications in microelectronics and life sciences. ${ }^{[5,6]}$ And while in the microelectronics industry the characteristic scale is dictated by the size of the transistors (approaching $10 \mathrm{~nm}$ ), in biology it is the micrometer-sized cell and its submicron compartments (nucleus, mitochondria, lysosomes and endosomes, etc.) which define the scale of interest.

Modern methods of cellular thermometry started with the creation of semiconductor quantum dots (QDs), that were able to produce both bright and stable fluorescence (Fig. 1). ${ }^{[7]}$ The latter turned out to be temperature-dependent, enabling their use as temperature sensors, ${ }^{[8,9]}$ and the size of QDs, ranging from single to tenths of nanometers, allowed them to penetrate tissues and cells ${ }^{[10]}$ easily. However, low biocompatibility of quantum dots due to their chemical composition (many of them were cadmium-based) decreases their chances of being used in vivo and to further advance in clinical applications. Another type of nanoparticle-based sensors - upconversion nanoparticles (UCNPs), containing rare-earth ions, while being more bio-compatible thanks to their core-shell structure, suffer from lower temperature resolution, ${ }^{[11-13]}$ which is not sufficient for exploring many intracellular processes.

The aforementioned biocompatibility issue can also be solved by using biomolecules, such as organic dyes, ${ }^{[14]}$ fluorescent polymers ${ }^{[15]}$ and fluorescent proteins. ${ }^{[16]}$ These molecules can be eas- 


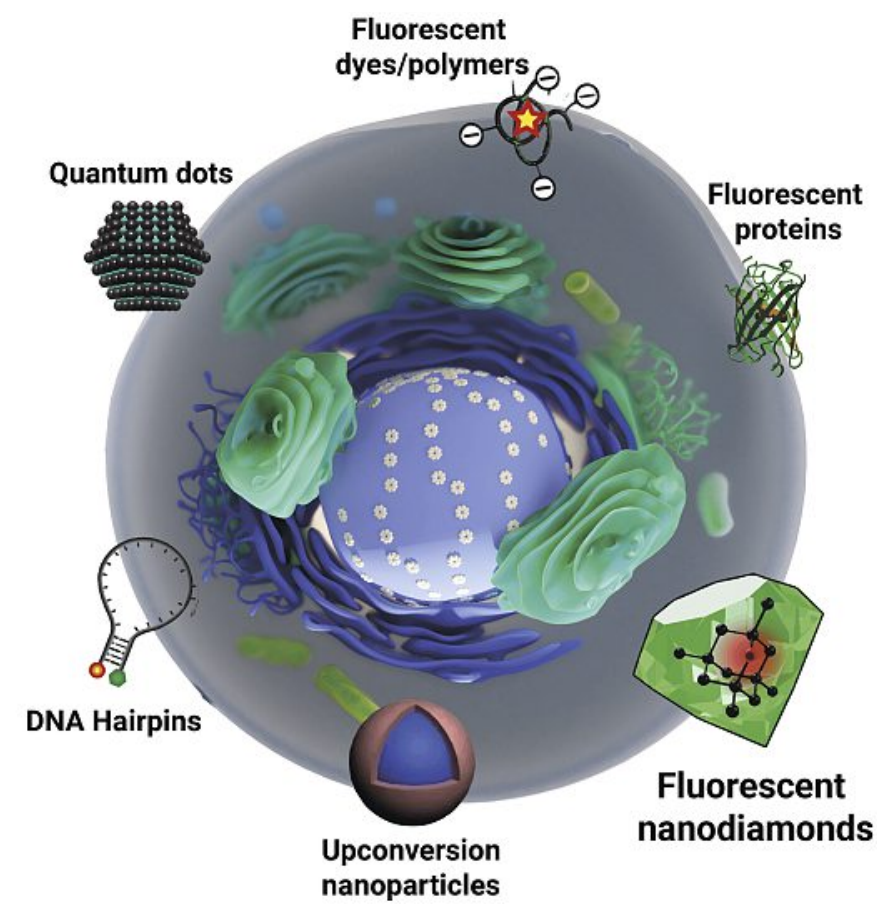

b)

\begin{tabular}{l|c|c|c} 
& Size (nm) & Accuracy (K) & References \\
\hline Quantum dots & $3-12$ & 0.2 & {$[8-9]$} \\
\hline $\begin{array}{l}\text { Upconversion } \\
\text { nanoparticles }\end{array}$ & $20-50$ & 0.3 & {$[11-13]$} \\
\hline $\begin{array}{c}\text { Fluorescent } \\
\text { dyes/polymers }\end{array}$ & $1-10$ & 0.3 & {$[14-15]$} \\
\hline $\begin{array}{c}\text { Fluorescent } \\
\text { proteins }\end{array}$ & $3-4$ & 0.4 & {$[16]$} \\
\hline \begin{tabular}{c} 
DNA Hairpins \\
\hline $\begin{array}{c}\text { Fluorescent } \\
\text { nanodiamonds }\end{array}$
\end{tabular} & $2-3$ & 0.5 & {$[19]$} \\
\hline
\end{tabular}

Fig. 1. Modern intracellular temperature sensors. a) Various types of commonly used non-invasive nanoscale temperature sensing techniques. b) A table listing the properties of the nanoscale temperature probes.

ily introduced into the cell, target various cell compartments and even be expressed by the cell machinery. Proof-of-principle experiments have shown the feasibility of such an approach, mapping sub-cellular temperature distribution and its dependence on the cell's metabolism. ${ }^{[14-17]}$ The inherent drawback of these techniques is in their susceptibility to the influence of the local chemical environment, ${ }^{[16,18,19]}$ which may alter the measured temperature. Being organic compounds, such fluorophores also suffer from unstable fluorescence and photobleaching, limiting their use in longer-term experiments. ${ }^{[20]}$

Fortunately, the ongoing search for new methods and techniques for nanoscale temperature sensing is constantly revealing new prospective candidates with superior sensing capabilities. ${ }^{[6,21]}$ Among them are fluorescent nanodiamonds (FNDs) - carbonbased bio-compatible nanoparticles, ${ }^{[22]}$ which produce bright and stable fluorescence due to the presence of various atomic impurities in their structure. ${ }^{[23]}$ In the next sections, we describe the main properties of FNDs, detail recent experiments utilizing them as temperature sensors and outline future perspectives in advancing their use for live-cell and in vivo experiments.

\section{Fluorescent Nanodiamonds}

Diamond has been recognized as a precious material for many centuries due to its remarkable physicochemical properties. However, only recently have scientists and engineers managed to create artificial diamonds using high-pressure high-temperature (HPHT) or chemical vapor deposition (CVD) methods, ${ }^{[24]}$ successfully controlling their size and chemical composition. ${ }^{[25]}$ This process is today not only revolutionizing the gemstone industry, ${ }^{[26]}$ but also opens a plethora of applications for diamonds in research. ${ }^{[27]}$ One example of such applications is quantum sensing using optically-active defects in diamond.[28]

Diamond has a cubic crystalline structure $\left(C_{3 \mathrm{v}}\right.$ symmetry) with an extremely high density $\left(1.77 \times 10^{23} \mathrm{~cm}^{-1}\right)$ and high Debye temperature $(2240 \mathrm{~K})$, which makes the diamond lattice an ideal host for hundreds of stable crystallographic defects. ${ }^{[23,29]}$ Many of these defects produce bright, continuous and non-blinking fluorescence in the visible and near-infrared range and are called 'color centers', as they give color to the normally colorless diamond gemstones. For example, the nitrogen-vacancy center, which is most commonly found in natural diamonds due to abundance of nitrogen in the atmosphere, makes the host diamond crystal appear yellowish. It is also the most-well studied diamond color center, ${ }^{[27]}$ which has been used to sense various physical parameters (magnetic ${ }^{[30]}$ and electric ${ }^{[31]}$ fields, $\mathrm{pH}^{[32]}$ strain, ${ }^{[33]}$ pressure $^{[34]}$ and temperature ${ }^{[35,36]}$ ) by utilizing its unique energy-level structure (Fig. 2). In recent years more and more experimentallyproduced diamond defects were recognized as prominent temperature sensors: silicon-, germanium-, tin- and nickel-vacancy centers. ${ }^{[37-40]}$ However, their production is still challenging and has to be upscaled before they can become a widely-used sensing tool (which is already the case for commercially-available ${ }^{[41]}$ nanodiamonds with NV-centers).

The obvious mechanism of temperature sensing in all the aforementioned color centers is based on the red shift of the zerophonon line (ZPL) in their emission spectra at an elevated temperature. ${ }^{[43]}$ The measurement setup for performing such temperature measurements therefore requires only an excitation laser and a spectrometer, and the achieved sensitivity approaches $0.3 \mathrm{~K}$ in $1 \mathrm{~s}$ of integration time at ambient temperature ${ }^{[37-39]}$ in both bulk diamond and nanodiamonds (100-200 nm in diameter). Although the simplicity of the technique makes it highly attractive, there are certain restrictions limiting its use, as the ZPL shift can be also influenced by different environmental parameters other than temperature. ${ }^{[39,44,45]}$ This limitation is especially severe in the case of the NV-centers, which are sensitive to the electric and magnetic field noise and may require individual pre-calibration for each emitter.

Fortunately, a special energy-level structure (spin-1 system) of NV-centers makes another measurement modality possible through the so-called optically-detected magnetic resonance (ODMR) technique. ${ }^{[46]}$ A simplified energy-level diagram in Fig. $2 \mathrm{c}$ shows a ground and an excited state (both triplets with radiative transitions between them) and a singlet meta-stable state through which the system can experience a non-radiative transition. At room temperature the sub-levels of both ground and excited states (having different spin projections $\mathrm{m}_{\mathrm{s}}=-1,0,1$ ) are non-degenerate, which makes the transitions between them possible (typically in the microwave range). Although the optical transitions from the ground to the excited state conserves the spin projection, the rate 
a)
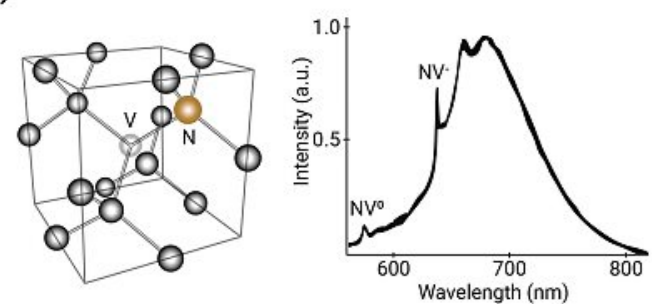

b)

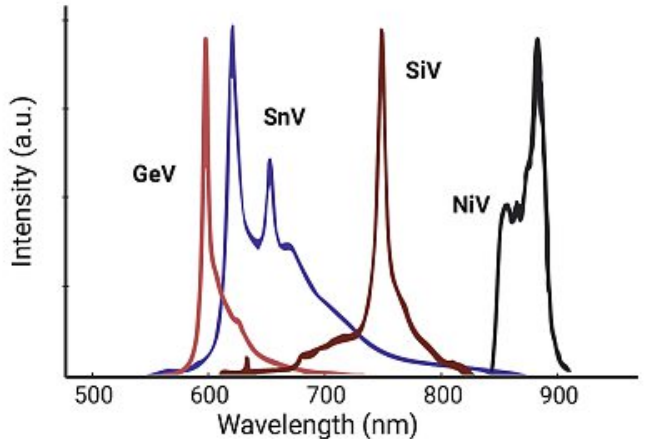

c)

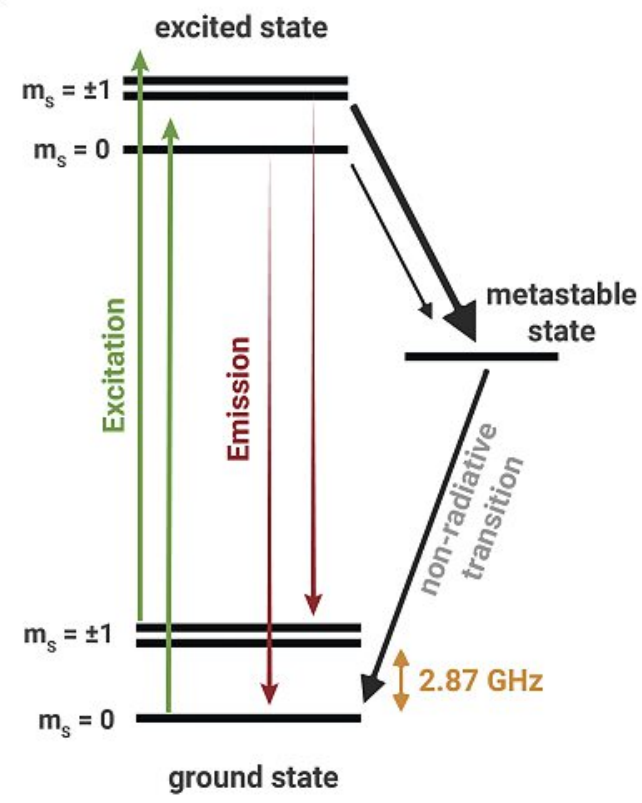

Fig. 2. Color centers in diamond, which can be used as a temperature sensor. a) Nitrogen-vacancy center, which can exist in various charge states $\left(\mathrm{NV}^{+}, \mathrm{NV}^{\circ}, \mathrm{NV}^{-}\right.$, where the $\mathrm{NV}^{-}$state is mostly used for sensing purposes) and its characteristic spectrum with two distinct peaks (zero-phonon lines) and a phonon sideband. (Adapted from ref. [42]) b) Spectra of other temperature-sensitive color centers. (Adapted from refs. [39,40]) c) Simplified energy-level structure of the $\mathrm{NV}^{-}$center. of transitions to the metastable state is much higher for the excited state with $\mathrm{m}_{\mathrm{s}}= \pm 1$ than for $\mathrm{m}_{\mathrm{s}}=0$, so the system will mostly relax to the ground state without emitting a photon. Normally, both sublevels are equally-populated, but if one applies a constant microwave signal at the transition frequency between them, this makes the $\mathrm{m}_{\mathrm{s}}= \pm 1$ states more populated (producing so-called spin polarization). More transitions therefore occur non-radiatively and fewer photons are detected as the fluorescence signal. Scanning the frequency of the microwaves around the transition, produces the ODMR graph shown in Fig. 3c.

As a change in temperature shifts the microwave frequency of the transition between the $\mathrm{m}_{\mathrm{s}}= \pm 1$ and $\mathrm{m}_{\mathrm{s}}=0$ sub-levels, ODMR can be used to measure relative temperature changes. ${ }^{[47]}$ Performing a straightforward calibration procedure converts these changes to the absolute temperature values. Using this method several research groups performed temperature measurements in a wide temperature range ${ }^{[13,35,48]}$ with a sensitivity approaching $100 \mathrm{mK}$ (NV-centers in nanodiamonds, $100 \mathrm{~nm}$ in diameter) and
$2 \mathrm{mK}$ (NV-centers in bulk diamond) for $1 \mathrm{~s}$ integration time, particularly in the biological environment and inside living cells. ${ }^{[35,49]}$ From the instrumentation point of view, an additional microwave source and an antenna to deliver microwaves in the vicinity of the $\mathrm{NV}$-center are required, but no spectrometer is needed in comparison to the previously described setup. Additional attention is also to be paid to two aspects: eliminating other influencing factors (e.g. varying magnetic field), which can be achieved by using specific microwave and laser pulse sequences, ${ }^{[35,50]}$ and avoiding the unwanted heating of the measured sample by microwaves, which in turn can be obtained by controlling dissipated power.

\section{Future Directions}

As one can see, the use of color centers in diamond for nanoscale temperature sensing is evolving rapidly and is currently at the stage where it is ready to be applied to the study of biological questions in living systems. All the aforementioned experiments, while being performed in fixed or even living cells, used an ex- a)

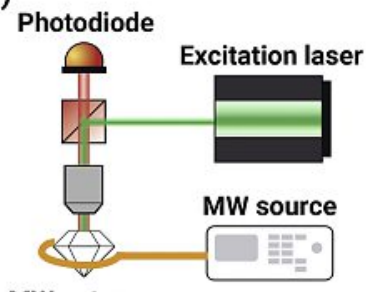

MW antenna b)

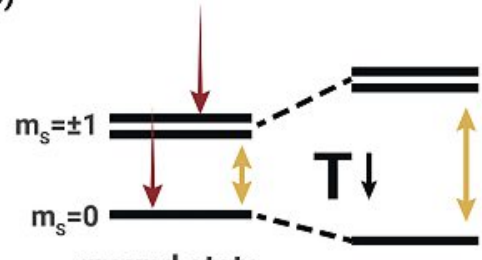

ground state c)

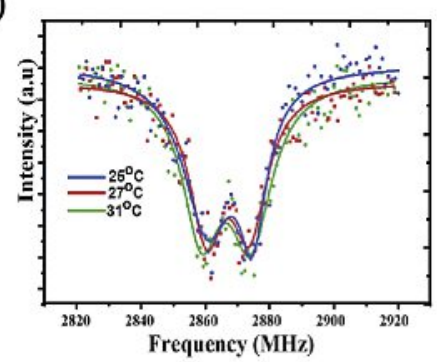

Fig. 3. Measurement schemes for temperature sensing using color centers in diamond. a-c) ODMR shift-based approach, $d$, e) ZPL shift-based approach. a, d) Simplified experimental setups, needed to perform, ODMR and ZPL-based temperature measurements, respectively. b) The shift of the energy sublevels in the ground state of the NV-center corresponding to the temperature change. c) Shift of the ODMR with temperature (adapted from ref. [13] with permission of AIP Publishing, https://doi. org/10.1063/1.5029904). e) Shift of the PL spectrum with temperature (adapted from ref. [37] with permission of OSA Publishing, https://doi.org/10.1364/ OL.42.004812). 
ternal laser to generate a local heat gradient (typically, through heating gold nanoparticles), that was later measured by a nearby color center. This is why the ultimate question is whether fluorescent nanodiamonds can measure local temperature differences and fluctuations in living cells, which occur naturally throughout their life cycle (for example, during ATP synthesis ${ }^{[14]}$ ).

To answer this question, not only is a high temperature sensitivity required, but also an ability to freely position nanodiamonds with sub-micron precision and to independently verify the measured temperature values. The verification can be performed by combining FNDs with other temperature sensors (e.g. UCNPs, metal nanoparticles, quantum dots $\left.{ }^{[13,51]}\right)$. Infrared imaging or miniaturized thermocouples can also be used for calibration and verification at the whole-cell level. Positioning nanodiamonds inside cells for high-resolution temperature mapping can be achieved in two ways: controlling the position of selected FNDs using external forces (e.g. thermophoretic force) or allowing nanodiamonds to attach themselves to only specific compartments of the cell, by modifying their surface with certain antigens. A measurement grid for temperature mapping could also be created by distributing a significant amount of FNDs inside the volume of the cell. However, the influence of the concentration of such nanoparticles in living cells on their normal functioning requires further exploration. ${ }^{[52]}$

Using smaller nanodiamonds together with super-resolution microscopy techniques can further increase the spatial resolution of temperature mapping ${ }^{[53-56]}$ and the increased number of color centers per diamond nanocrystal allows the temporal resolution to be improved (faster acquisition time for the same sensitivity). ${ }^{[35]}$ Undoubtedly, in parallel with the development of nanothermometers, based on the currently-known color centers, new promising color centers will continue to appear and be explored for sensing applications. ${ }^{[40]}$ This applies not only to optically-active defects in diamonds, but also in other materials, like silicon carbide, ${ }^{[57]}$ or even 2D materials (e.g. h-BN flakes $\left.{ }^{[58,59]}\right)$.

Finally, we could foresee the creation of multifunctional nanodiamond-based sensors, capable of measuring various physicochemical properties inside living cells with an unprecedented resolution. This can be achieved by either combining different color centers within the same diamond nanocrystal or coupling together several nanoparticles with different sensing functionalities. ${ }^{[21,60]}$ Just one more step is then needed to turn such nanoscale sensors into fully-functional theranostic agents which, depending on the local environment, can controllably release drug molecules ${ }^{[61]}$ and measure the dynamical reaction of the intracellular environment to them.

\section{Acknowledgments}

We thank the financial support from EPFL Lausanne. This research was also partly supported by the Swiss National Science Foundation through the National Centre of Competence in Research Bio-Inspired Materials.

Received: November 21, 2018

[1] M. Bell, Math. Teach. 1937, 30, 80

[2] C. Wunderlich, 'On the temperature in disease, a manual of medical thermometry', The New Sydenham Society, London, 1871.

[3] Y. Yue, X. Wang, Nano Rev. 2012, 3, 11586, doi: 10.3402/nano.v3i0.11586.

[4] K. M. Mccabe, M. Hernandez, Pediatr. Res. 2010, 67, 469, doi: 10.1203/ PDR.0b013e3181d68cef.Molecular.

[5] X. D. Wang, O. S. Wolfbeis, R. J. Meier, Chem. Soc. Rev. 2013, 42, 7834, doi: $10.1039 / \mathrm{c} 3 \operatorname{cs} 60102 \mathrm{a}$

[6] T. Bai, N. Gu, Small 2016, 12, 4590, doi: 10.1002/smll.201600665.

[7] S. Wang, S. Westcott, W. Chen, J. Phys. Chem. B 2002, 106, 11203, doi: $10.1021 / \mathrm{jp} 026445 \mathrm{~m}$.

[8] L. M. Maestro, E. M. Rodríguez, F. S. Rodríguez, M. C. I. De La Cruz, A. Juarranz, R. Naccache, F. Vetrone, D. Jaque, J. A. Capobianco, J. G. Solé, Nano Lett. 2010, 10, 5109, doi: 10.1021/n11036098.
[9] L. M. Maestro, P. Haro-Gonzalez, M. C. Iglesias-De La Cruz, F. SanzRodriguez, A. Juarranz, J. G. Sole, D. Jaque, Nanomedicine 2013, 8, 379, doi: $10.2217 / \mathrm{nnm} .12 .122$.

[10] H. Liu, Y. Fan, J. Wang, Z. Song, H. Shi, R. Han, Nat. Publ. Gr. 2015, 1, doi: $10.1038 /$ srep 14879 .

[11] G. Jiang, S. Zhou, X. Wei, Y. Chen, C. Duan, M. Yin, B. Yang, W. Cao, RSC Adv. 2016, 6, 11795, doi: 10.1039/C5RA27203C.

[12] T. V. Gavrilović, D. J. Jovanović, V. Lojpur, M. D. Dramićanin, Sci. Rep. 2014, 4, 1, doi: 10.1038/srep04209.

[13] M. Alkahtani, L. Jiang, R. Brick, P. Hemmer, M. Scully, Opt. Lett. 2017, 42, 4812, doi: 10.1364/OL.42.004812.

[14] D. Chrétien, P. Bénit, H. H. Ha, S. Keipert, R. El-Khoury, Y. T. Chang, M. Jastroch, H. T. Jacobs, P. Rustin, M. Rak, PLoS Biol. 2018, 16, 1, doi: 10.1371/journal.pbio.2003992.

[15] K. Okabe, N. Inada, C. Gota, Y. Harada, T. Funatsu, S. Uchiyama, Nat. Commun. 2012, 3, 705, doi: 10.1038/ncomms1714.

[16] J. Donner, S. Thompson, M. Kreuzer, G. Baffou, J. Donner, S. Thompson, M. Kreuzer, G. Baffou, R. Quidant, Nano Lett. 2012, 12, 2107, doi: 10.1021/ nl300389y.

[17] S. Kiyonaka, T. Kajimoto, R. Sakaguchi, D. Shinmi, M. Omatsu-Kanbe, H. Matsuura, H. Imamura, T. Yoshizaki, I. Hamachi, T. Morii, Y. Mori, Nat. Methods 2013, 10, 1232, doi: 10.1038/nmeth.2690.

[18] F. Vetrone, R. Naccache, A. Zamarrón, A. Juarranz de la Fuente, F. SanzRodríguez, L. Martinez Maestro, E. Martín Rodriguez, D. Jaque, J. García Solé, J. a Capobianco, ACS Nano 2010, 4, 3254, doi: 10.1021/nn100244a.

[19] A. T. Jonstrup, J. Fredsøe, A. H. Andersen, Sensors 2013, 13, 5937, doi: 10.3390/s130505937.

[20] D. Jaque, B. Del Rosal, E. M. Rodríguez, L. M. Maestro, P. Haro-González, J. G. Solé, Nanomedicine 2014, 9, 1047, doi: 10.2217/nnm.14.59.

[21] S. Sotoma, C. P. Epperla, H. C. Chang, ChemNanoMat 2018, 4, 15, doi: 10.1002/cnma.201700257.

[22] L. P. Mcguinness, Y. Yan, A. Stacey, D. A. Simpson, L. T. Hall, D. Maclaurin, S. Prawer, P. Mulvaney, J. Wrachtrup, F. Caruso, R. E. Scholten, L. C. L. Hollenberg, Nat. Nanotechnol. 2011, 6, 358, doi: 10.1038/nnano.2011.64.

[23] I. Aharonovich, S. Castelletto, D. A. Simpson, C. H. Su, A. D. Greentree, S. Prawer, Reports Prog. Phys. 2011, 74, doi: 10.1088/0034-4885/74/7/076501.

[24] R. S. Balmer, J. R. Brandon, S. L. Clewes, H. K. Dhillon, J. M. Dodson, I. Friel, P. N. Inglis, T. D. Madgwick, M. L. Markham, T. P. Mollart, J. Phys. Condens. Matter 2009, 21, 364221, doi: 10.1088/0953-8984/21/36/364221.

[25] V. N. Mochalin, O. Shenderova, D. Ho, Y. Gogotsi, Nat. Nanotechnol. 2012, 7, 11, doi: 10.1038/nnano.2011.209.

[26] T. W. Overton, J. E. Shigley, Gems Gemol. 2008, 44, 32, doi: 10.1057/9781137537614_8.

[27] R. Schirhagl, K. Chang, M. Loretz, C. L. Degen, Phys. Chem. 2014, 65, 83, doi: 10.1146/annurev-physchem-040513-103659.

[28] Y. Wu, F. Jelezko, M. B. Plenio, T. Weil, Angew. Chem. Int. Ed. 2016, 55, 6586, doi: 10.1002/anie.201506556.

[29] A. M. Zaitsev, 'Optical properties of diamond: a data handbook', Springer Science \& Business Media, 2013.

[30] G. Balasubramanian, I. Y. Chan, R. Kolesov, M. Al-Hmoud, J. Tisler, C. Shin, C. Kim, A. Wojcik, P. R. Hemmer, A. Krueger, T. Hanke, A. Leitenstorfer, R. Bratschitsch, F. Jelezko, J. Wrachtrup, Nature 2008, 455, 648, doi: 10.1038/ nature 07278

[31] F. Dolde, H. Fedder, M. W. Doherty, T. Nöbauer, F. Rempp, G. Balasubramanian, T. Wolf, F. Reinhard, L. C. L. Hollenberg, F. Jelezko, J. Wrachtrup, Nat. Phys. 2011, 7, 459, doi: 10.1038/nphys 1969.

[32] V. Petrakova, I. Rehor, J. Stursa, M. Ledvina, M. Nesladek, P. Cigler, Nanoscale 2015, 7, 12307, doi: 10.1039/c5nr00712g.

[33] J. R. Maze, A. Gali, E. Togan, Y. Chu, A. Trifonov, E. Kaxiras, M. D. Lukin, New J. Phys. 2011, 13, 1, doi: 10.1088/1367-2630/13/2/025025.

[34] M. W. Doherty, V. V Struzhkin, D. A. Simpson, L. P. Mcguinness, Y. Meng, T. J. Karle, R. J. Hemley, N. B. Manson, L. C. L. Hollenberg, S. Prawer, Phys. Rev. Lett. 2014, 112, 047601, doi: 10.1103/PhysRevLett.112.047601.

[35] G. Kucsko, P. C. Maurer, N. Y. Yao, M. Kubo, H. J. Noh, P. K. Lo, H. Park, M. D. Lukin, Nature 2013, 500, 54, doi: 10.1038/nature12373.

[36] D. M. Toyli, C. F. de las Casas, D. J. Christle, V. V Dobrovitski, D. D. Awschalom, C. F. De, D. David, Proc. Natl. Acad. Sci. USA 2013, 110, 8417, doi: 10.1073/pnas.1306825110.

[37] C. T. Nguyen, R. E. Evans, A. Sipahigil, M. K. Bhaskar, D. D. Sukachev, V. N. Agafonov, V. A. Davydov, L. F. Kulikova, F. Jelezko, M. D. Lukin, Appl. Phys. Lett. 2018, 112, doi: 10.1063/1.5029904.

[38] J.-W. Fan, I. Cojocaru, J. Becker, I. V. Fedotov, M. H. A. Alkahtani, A Alajlan, S. Blakley, M. Rezaee, A. Lyamkina, Y. N. Palyanov, Y. M. Borzdov, Y.-P. Yang, A. Zheltikov, P. Hemmer, A. V. Akimov, ACS Photonics 2018, 5 , 765, doi: 10.1021/acsphotonics.7b01465.

[39] M. Alkahtani, I. Cojocaru, X. Liu, T. Herzig, J. Meijer, J. Küpper, T. Lühmann, A. V Akimov, P. R. Hemmer, M. Alkahtani, I. Cojocaru, X. Liu, T. Herzig, J. Meijer, Appl. Phys. Lett. 2018, 241902, doi: 10.1063/1.5037053.

[40] M. H. Alkahtani, F. Alghannam, L. Jiang, A. A. Rampersaud, R. Brick, C. L. Gomes, M. O. Scully, P. R. Hemmer, Opt. Lett. 2018, 43, 3317, doi: 10.1364/ OL.43.003317. 
[41] O. Shenderova, N. Nunn, T. Oeckinghaus, M. Torelli, G. Mcguire, K. Smith, E. Danilov, R. Reuter, J. Wrachtrup, A. Shames, D. Filonova, A. Kinev, A. Nanotechnologies, Proc. SPIE, Adv. Photonics Quantum Comput. Mem. Commun. X 2017, 10118, doi: 10.1117/12.2256800.

[42] I. Aharonovich, S. Castelletto, D. A. Simpson, C.-H. Su, A. D. Greentree, S. Prawer, Reports Prog. Phys. 2011, 74, 076501, doi: 10.1088/00344885/74/7/076501.

[43] K. D. Jahnke, A. Sipahigil, J. M. Binder, M. W. Doherty, M. Metsch, L. J Rogers, N. B. Manson, M. D. Lukin, F. Jelezko, New J. Phys. 2015, 17, doi: 10.1088/1367-2630/17/4/043011.

[44] P.-C. Tsai, C. P. Epperla, J.-S. Huang, O. Y. Chen, C.-C. Wu, H.-C. Chang, Angew. Chemie Int. Ed. 2017, 56, 3025, doi: 10.1002/anie.201700357.

[45] T. Plakhotnik, H. Aman, H. C. Chang, Nanotechnology 2015, 26, doi: 10.1088/0957-4484/26/24/245501.

[46] A. Gruber, A. Dra, C. Tietz, L. Fleury, J. Wrachtrup, C. Von Borczyskowski, Science 1997, 276, 2012, doi: 10.1126/science.276.5321.2012.

[47] V. M. Acosta, E. Bauch, M. P. Ledbetter, A. Waxman, L. S. Bouchard, D. Budker, Phys. Rev. Lett. 2010, 104, 1, doi: 10.1103/PhysRevLett.104.070801.

[48] P. Neumann, I. Jacobi, F. Dolde, C. Burk, R. Reuter, G. Waldherr, J. Honert, T. Wolf, A. Brunner, J. H. Shim, D. Suter, H. Sumiya, J. Isoya, J. Wrachtrup, Nano Lett. 2013, 13, 2738, doi: 10.1021/nl401216y.

[49] S. Takeharu, S. Sotoma, Y. Harada, Biophys. Physicobiology 2018, 15, 151, doi: 10.2142/biophysico.15.0.

[50] P. Konzelmann, T. Rendler, V. Bergholm, A. Zappe, V. Pfannenstill, M. Garsi, F. Ziem, M. Niethammer, M. Widmann, S.-Y. Lee, P. Neumann, J. Wrachtrup, arXiv 2018, 1808.04087.

[51] W. Liu, B. Naydenov, S. Chakrabortty, B. Wuensch, K. Hübner, S. Ritz, H. Cölfen, H. Barth, K. Koynov, H. Qi, R. Leiter, R. Reuter, J. Wrachtrup, F. Boldt, J. Scheuer, U. Kaiser, M. Sison, T. Lasser, P. Tinnefeld, F. Jelezko,
P. Walther, Y. Wu, T. Weil, Nano Lett. 2016, 16, 6236, doi: 10.1021/acs. nanolett.6b02456.

[52] A. M. Schrand, H. Huang, C. Carlson, J. J. Schlager, E. Osawa, S. M. Hussain, L. Dai, J. Phys. Chem. B 2007, 111, 2, doi: 10.1021/jp066387v.

[53] M. Gu, Y. Cao, S. Castelletto, B. Kouskousis, X. Li, Opt. Express 2013, 21, doi: 10.1364/OE.21.017639.

[54] X. Yang, Y.-K. Tzeng, Z. Zhu, Z. Huang, X. Chen, Y. Liu, H.-C. Chang, L. Huang, W.-D. Li, P. Xi, RSC Adv. 2014, 4, 11305, doi: 10.1039/C3RA47240J.

[55] Y. K. Tzeng, O. Faklaris, B. M. Chang, Y. Kuo, J. H. Hsu, H. C. Chang, Angew. Chem. Int. Ed. 2011, 50, 2262, doi: 10.1002/anie.201007215.

[56] S. Sotoma, D. Terada, T. F. Segawa, R. Igarashi, Y. Harada, M. Shirakawa, Sci. Rep. 2018, 8, 1, doi: 10.1038/s41598-018-23635-5.

[57] H. Kraus, V. A. Soltamov, F. Fuchs, D. Simin, A. Sperlich, P. G. Baranov, G. V Astakhov, V. Dyakonov, Sci. Rep. 2014, 4, doi: 10.1038/srep05303.

[58] J. Feng, H. Deschout, S. Caneva, S. Hofmann, I. Lončarić, P. Lazić, A. Radenovic, Nano Lett. 2018, 18, 1739.

[59] N. R. Jungwirth, B. Calderon, Y. Ji, M. G. Spencer, M. E. Flatte, G. D. Fuchs, Nano Lett. 2016, 16, 6052, doi: 10.1021/acs.nanolett.6b01987.

[60] N. Wang, G. Q. Liu, W. H. Leong, H. Zeng, X. Feng, S. H. Li, F. Dolde, H. Fedder, J. Wrachtrup, X. D. Cui, S. Yang, Q. Li, R. B. Liu, Phys. Rev. X 2018, 8, doi: 10.1103/PhysRevX.8.011042.

[61] X. Chen, W. Zhang, Chem. Soc. Rev. 2017, 46, 734, doi: 10.1039/c6cs00109b. 